\title{
DIVISIÓN INTRAINDUSTRIAL DEL TRABAJO EN LAS ENTIDADES FEDERATIVAS DE MÉXICO*
}

\author{
HARLEY L. Browning y JACK P. GibBS \\ Population Research Center, \\ Universidad de Texas
}

\begin{abstract}
Aunoue el estudio clásico de Durkheim (1949) estableció la importancia sociológica de la división del trabajo, no creó una tradición en la investigación. En verdad, su trabajo es citado con frecuencia pero hasta hace poco los sociólogos no se han ocupado de la investigación sistemática de la división del trabajo y varios aspectos del fenómeno aún están en gran medida inexplorados. En particular, la investigación ha enfocado las tendencias históricas y las diferencias internacionales, pero la división del trabajo también varía marcadamente entre las industrias y las regiones de un mismo país.

Este trabajo se ocupa de la división intraindustrial del trabajo en las entidades de México. La investigación está vinculada tanto al trabajo de Durkheim como a la noción de desarrollo económico. La mayoría de las investigaciones y conceptualizaciones del desarrollo económico considera a los países como unidades, pero el desarrollo no es nunca uniforme intranacionalmente. Las diferencias entre regiones son tan grandes que son conocidas como aspectos de un "dualismo" en el desarrollo económico (ver Higgins, 1968; Hirschman, 1958).
\end{abstract}

\section{Conceptualización}

Ni Durkheim ni Adam Smith, investigadores precursores, se preocuparon por el problema de analizar la división del trabajo, y aún hoy el concepto es ambiguo. El problema principal es que los sociólogos y los economistas no han logrado distinguir entre las bases y el grado de la división del trabajo. El grado es el monto de diferencia que existe entre individuos en sus labores de sustento, y las bases se refieren a los correlativos de dichas diferencias individuales. Por ejemplo, si los individuos de una edad particular se ocupan de un tipo de labor de sustento que les es peculiar, entonces la edad es una base de la división del trabajo. Similarmente, siempre que el lugar de trabajo o residencia y el tipo de labor de sustento estén relacionados, la división del trabajo tiene una base territorial.

* E1 título original de este trabajo es "Intraindustry Division of Labor: The States of Mexico", y fue llevado a cabo en el Centro de Investigación de la Población de la Universidad de Texas, Austin, y apoyado en parte por el Instituto de Estudios Latinoamericanos de la misma Universidad. La traducción al español es de Brígida García. 
Dada la tendencia a pensar en la división del trabajo en términos de ocupaciones o industrias, la referencia que se ha hecho a las actividades de sustento necesita explicarse. Una labor de sustento es un gasto de energía humana en la búsqueda directa de alimento o en la producción de algún bien o servicio. Dichas labores constituyen el referente fundamental en el análisis de la división del trabajo porque son la base de las distinciones ocupacionales e industriales. Es verdad que debido sólo a consideraciones prácticas, la investigación sobre división del trabajo debe estar basada en estadísticas ocupacionales e industriales, pero la diferenciación entre las labores de sustento es sin embargo fundamental. Ese punto es tanto más importante porque puede no ser factible analizar la división del trabajo en poblaciones analfabetas haciendo referencia a ocupaciones o industrias. En esas poblaciones los individuos tienden a saber "hacerlo todo", y sus labores de sustento varían estacionalmente o aun por hora en tal medida que los conceptos de ocupación e industria son raramente aplicables (Herskovits, 1952).

La división del trabajo ocasiona tanto una diferenciación en las labores de sustento como un intercambio relacionado de bienes y servicios. Sin embargo, se supone que la interdependencia funcional y la diferenciación van de la mano (ver Hawley, 1950), lo que significa que la proporción de un componente variaría directamente en la proporción del otro. Este supuesto es necesario ya que para ciertos tipos de investigación no es fácil encontrar datos disponibles comparables y adecuados sobre el volumen de bienes y servicios. Por consiguiente, el primer paso al combinar la división del trabajo a las referencias empíricas es el Planteamiento I: a mayor división del trabajo corresponde una mayor diferencia entre individuos en sus labores de sustento. Este planteamiento no debe ser interpretado como una definición; más bien, establece una relación directa entre los dos componentes de la división del trabajo.

Como sugiere lo anterior, la división del trabajo es considerada como una estructura hipotética y no, al contrario de los tratamientos anteriores (Gibbs y Martin, 1962; Gibbs y Browning, 1966) como algo directamente medible. Lo mismo puede afirmarse de la segunda variable en el planteamiento I: en la actualidad no hay forma de comparar poblaciones con respecto al monto de diferenciación en sus labores de sustento. Las estadísticas requeridas para dichas comparaciones no son tabuladas en ningún país, y tan sólo el costo desanima la recolección de los datos requeridos a través de encuestas.

Aun cuando la diferenciación en las actividades de sustento no es mensurable, se supone que se refleja en las distinciones ocupacionales. Ese supuesto se establece formalmente en el Planteamiento II : cuanto mayor sea la diferencia entre individuos en sus labores de sustento, mayor será la diferenciación ocupacional. Los planteamientos I y II suministran la base para derivar la relación entre la división del trabajo como una estructura y la diferenciación ocupacional en el Planteamiento III : cuanto mayor sea el grado de división del trabajo, mayor será la diferenciación ocupacional. 


\section{MEDIDAS DE DIFERENCIACIÓN OCUPACIONAL}

Por definición, la diferenciación ocupacional es función del número de ocupaciones y de la distribución de los individuos entre las ocupaciones. Obviamente, con una sola ocupación no hay diferenciación, pero la diferenciación también entraña la distribución de los individuos por categorías. Para ilustrar esto, consideremos una población en la cual existen 500 ocupaciones. Este número sugiere por sí sólo una diferenciación considerable, pero las diferencias en las labores de sustento se minimizarán si casi todos los individuos estuvieran concentrados en una ocupación.

Dada la anterior conceptualización de diferenciación ocupacional (de aquí en adelante $D O$ ) una medida apropiada es $D O=1-\left[\sum X^{2} /\right.$ $\left.(\Sigma X)^{2}\right]$ donde $X$ es el número de individuos en cada ocupación. Una ventaja de esta medida es que un valor de $D O$ refleja tanto el número de ocupaciones como la distribución de los individuos. Por ejemplo, con 4 ocupaciones y una distribución uniforme entre ellas, el valor de $D O$ es .750 , pero con cinco ocupaciones y una distribución uniforme es .800 . Otra ventaja de la medida es su ámbito de aplicación. Un valor $D O$ no es función del tamaño de la población, y esa característica hace posible la comparación de los valores $D O$ de las poblaciones sin considerar la variación en el tamaño de la fuerza de trabajo. Además, la medida puede ser aplicada a cualquier clase de población y a otras categorías no ocupacionales. Se aplica a industrias en la misma forma que a las ocupaciones y puede ser usada para medir la diferenciación ocupacional dentro de una industria. Finalmente, aunque la lista de ocupaciones o industrias debe ser detallada y completa en cada población, la medida de diferenciación no necesita basarse en la misma lista en todas las poblaciones.

Aunque la medida anterior tiene algunas propiedades convenientes, no está libre de limitaciones o críticas, una de las cuales es matemática. La medida no da suficiente ponderación al número de ocupaciones, como cuando se consideran los siguientes valores máximos de $D O$ con varios números de ocupaciones: dos ocupaciones, .500; cuatro ocupaciones, .750; y ocho ocupaciones, .875. Obsérvese que al duplicar el número de ocupaciones el valor de $D O$ no cambia proporcionalmente aun cuando la distribución de los individuos es una constante.

\section{MÉxico COMO UN CASO DE DESARROLlO REgIONAL DESIGUAL}

En 1930 México era claramente un país subdesarrollado con las dos terceras partes de su población analfabeta, dos terceras partes de su fuerza de trabajo en la agricultura y sólo cerca de un quinto de su población en zonas urbanas de 10000 o más habitantes. En contraste, se espera que los resultados del censo de 1970 muestren un alfabetismo de más del $70 \%$, menos de la mitad de la fuerza de trabajo en la agricultura y cerca de $50 \%$ de la población en zonas urbanas de 10000 y más habitantes.

Si bien el desarrollo económico de México ha sido sostenido e impresionante en los últimos tres decenios, también ha sido desigual. 
Algunas entidades, especialmente aquellas con población indígena numerosa y agricultura de subsistencia, han participado poco en el auge económico. La urbanización refleja este desarrollo desigual. Por ejemplo, en 1960 las tres entidades mexicanas del sur (Oaxaca, Chiapas y Guerrero) tenían menos del $15 \%$ de su población viviendo en localidades urbanas de 10000 y más habitantes, mientras que las tres entidades del nordeste (Tamaulipas, Nuevo León y Coahuila) tenían más del $50 \%$ de su población en tales lugares. En la actualidad pocos países del mundo tienen tal diversidad socioeconómica regional como México. (El mejor tratamiento de las diferencias regionales mexicanas continúa siendo el de Yates, 1961). Véanse también los de Bataillon, 1969, para las diferencias geográficas; Unikel, 1970, para las urbanas, y Mir, 1970, para las educativas.

\section{DIFERENCIACIÓN OCUPACIONAL DENTRO DE LAS INDUSTRIAS, POR ENTIDADES}

Los censos de 1950 y 1960 suministran datos para medidas de diferenciación ocupacional intraindustrial en cada una de las 32 entidades mexicanas. Las medidas son ilustradas en el cuadro 1, donde ocho categorías industriales y nueve categorías ocupacionales se clasifican en forma cruzada para Jalisco, uno de los estados mexicanos más grandes y más diversificados.

Tanto las categorías industriales como las ocupacionales son muy gruesas, pero se harán comentarios más amplios sobre este punto en la interpretación de los resultados. Por otra parte, ya que las categorías ocupacionales son constantes entre las industrias, un valor de $D O$ en el cuadro 1 refleja solamente la distribución de los individuos. Con nueve categorías ocupacionales el valor máximo es .889 , lo cual indica un número igual de individuos en cada categoría ocupacional de la industria.

Se observa que algunos de los elementos del cuadro son ceros debido a las distribuciones censales mexicanas. Por ejemplo, no hay administradores, directores, o "trabajadores" en la agricultura. Nótese también que la denominación de trabajadores (obreros) no es exactamente una distinción entre obreros calificados, semi-calificados o no calificados en la forma que existe en los Estados Unidos. La diferencia en calificación está presente, pero estrictamente hablando la distinción es entre "trabajadores" y "ayudantes".

La división del trabajo es un componente del desarrollo económico y, dada la relación teórica entre éste y la diferenciación ocupacional, las cifras del cuadro 2 sugieren claramente que el desarrollo económico es marcadamente diferente entre las entidades mexicanas. Para algunas industrias el valor de $D O$ en una entidad está muy por encima del doble del valor en otra entidad.

Si la medida $D O$ está relacionada con el desarrollo económico, debería deducirse que entre 1950 y 1960, decenio de indiscutible crecimiento económico en México, esta medida debería aumentar en todas las industrias en todas las entidades. En total, esta perspectiva se confirma en el $75 \%$ de los 256 casos ( 8 industrias en cada una de las 32 entidades). El número de predicciones acertadas por industria es: agri- 
cultura, 32 ; minería, 23 ; manufacturas, 31 ; construcción, 22 ; electricidad, gas, etc., 21 ; comercio, 29 ; transportes, 4 ; y servicios, 29 . Los transportes constituyen la más notoria excepción y cuando se le omite del total, el porciento de predicciones acertadas sube a 84 . Mientras que en otras industrias los descensos en $D O$ son insignificantes, en los transportes el descenso es típicamente considerable (v.g.: de .615 a .466 en Sinaloa). No hay una explicación aceptable para la magnitud y consistencia del descenso en los transportes, pero puede representar un cambio en las distribuciones censales en vez de cambios reales en la estructura ocupacional de esa industria.

La interpretación de los valores $D O$ en términos tanto de diferencias entre las industrias como de diferencias entre las entidades es una tarea complicada. En general, se supone que los valores $D O$ varían inversamente con respecto a la productividad por trabajador. Por ejemplo, con una excepción (el casi completamente urbano Distrito Federal) los valores $D O$ para la agricultura son bajos en todas las entidades. Los valores bajos se deben en parte a la naturaleza de la organización agrícola mexicana, y en parte a las distribuciones censales mexicanas. La minería es quizás la única excepción a la idea de que un valor bajo de $D O$ refleja baja productividad. Las más importantes entidades mineras de México (Chihuahua, Coahuila, Durango, Hidalgo, San Luis Potosí y Zacatecas) tienen valores de $D O$ bajos, lo cual no quiere decir que la productividad sea baja, ya que en estos estados se explota la minería en gran escala. En contraste, entidades altamente urbanizadas como el Distrito Federal, Nuevo León y Baja California Norte tienen pocas operaciones en gran escala. En estos grandes contextos urbanos la "minería" se limita a la explotación de canteras, arena y otras actividades relacionadas con la construcción. Estas actividades emplean un número considerable de trabajadores de oficina y de transporte, lo cual hace que el valor de $D O$ suba para esta industria.

Las manufacturas tienen un margen de fluctuación considerable que refleja las grandes diferencias entre los diversificados centros manufactureros del país, como el caso del Distrito Federal ( $D O$ de .526) y Tlaxcala,, un estado económicamente atrasado que depende de una organización manufacturera textil y artesanal anticuada ( $D O$ de $.214)$.

Los valores de $D O$ para la construcción, electricidad, gas, etc., y los servicios varían poco entre las entidades, aun cuando las causas no son las mismas para las tres industrias. En México, la construcción es todavía intensiva en trabajo aun en áreas metropolitanas debido a la gran oferta de mano de obra no calificada y mal remunerada (los inmigrantes de áreas rurales constituyen gran parte de este grupo) y debido a que las técnicas de construcción permiten el uso de este tipo de mano de obra aun en edificios de diseño moderno. Por lo tanto, hay relativamente poca variación de entidad a entidad en los valores de $D O$ para la construcción. En el caso de la electricidad, gas, etc., la poca fluctuación se debe a la aplicación relativamenie uniforme de tecnología avanzada. Las plantas generadoras de energía eléctrica y los sistemas de aguas y alcantarillado no se prestan a una modernización "parcial". Además, estos sistemas a menudo son establecidos en el 
Cuadro 1

JALISCO: DistRIBUCiÓN DE LA FUERZA DE TRABAJO ENTRE OCUPACIONES POR INDUSTRIAS Y DIFERENCIACIÓN OCUPACIONAL INTRAINDUSTRIAL, 1960

\begin{tabular}{|c|c|c|c|c|c|c|c|c|}
\hline \multirow{2}{*}{ Ocupaciones } & \multicolumn{8}{|c|}{$I \quad n \quad d \quad t \quad x$ a $B$} \\
\hline & Agricul tura & Minerla & Manufacturas & Construceion & $\begin{array}{l}\text { Electricidad, } \\
\text { Eas, oto. }\end{array}$ & Comercio & Transportes & Servicios \\
\hline $\begin{array}{l}\text { Profesionis } \\
\text { tas }\end{array}$ & 1319 & 770 & 3406 & 1493 & 186 & 2125 & 477 & 18976 \\
\hline $\begin{array}{l}\text { Adminis trado } \\
\text { ros y di- } \\
\text { rectores }\end{array}$ & 0 & 96 & 2348 & 359 & 70 & 1450 & 207 & 1388 \\
\hline Oficinistas & 1144 & 629 & 5827 & 573 & 761 & 6129 & 3472 & 17534 \\
\hline Vendedores & 2924 & 206 & 3638 & 125 & 21 & 71361 & 1136 & 4083 \\
\hline Agricul tures & 387082 & 0 & 0 & 0 & 0 & 0 & 0 & 0 \\
\hline Mineros & 0 & 2983 & 0 & 0 & 0 & 0 & 0 & 0 \\
\hline $\begin{array}{l}\text { Trabajadores } \\
\text { Califica- } \\
\text { dos }\end{array}$ & 0 & 0 & 93391 & 24401 & 1194 & 1531 & 16575 & 3019 \\
\hline $\begin{array}{l}\text { Trabajadores } \\
\text { no califi- } \\
\text { cados y se } \\
\text { mi-oalifi- } \\
\text { cados }\end{array}$ & 0 & 0 & 7994 & 6396 & 186 & 832 & 2540 & 3187 \\
\hline $\begin{array}{l}\text { Trabajadores } \\
\text { en servicios }\end{array}$ & 647 & 103 & 1809 & 339 & 50 & 617 & 316 & 43529 \\
\hline $\begin{array}{l}\text { Diferenciación } \\
\text { ocupaoional } \\
\text { intraindus- } \\
\text { trial } \\
1-\left[\left[\mathbf{x}^{2} /\left([\mathbf{x})^{2}\right] \mathrm{a} /\right.\right.\end{array}$ & .030 & .566 & .369 & .437 & .658 & .272 & .518 & 691 \\
\hline
\end{tabular}

Fuente: Estados Unidos Mexicanos, VIII Censo General de Población-1960, Estado de Jalisco, 1963, cuadro 22 
país por agencias del gobierno central, las que por lo general tienen normas uniformes. El tercer caso, los servicios, tiene un valor $D O$ alto en todas las entidades porque incluyen una diversidad de subindustrias heterogéneas. En las entidades en que se emplean muchos profesionales hay una gran probabilidad de un gran número de sirvientes.

Aunque la evidencia de tasas divergentes de desarrollo económico entre las entidades mexicanas no debe extrañar, eso no indica que la variación en la estructura ocupacional e industrial sea una demostración superflua. Desde luego que algunos aspectos de la estructura ocupacional e industrial no son necesariamente indicadores de desarrollo económico divergente a nivel intranacional. Una vez que el desarrollo económico alcanza cierta magnitud, algunas divisiones territoriales de una nación pueden especializarse en la producción de bienes y servicios particulares y esa especialización tiende a disminuir la diferenciación ocupacional e industrial en ellas. Por ejemplo, mientras que la proporción de personas empleadas en manufacturas puede ser un indicador de desarrollo a nivel nacional, el hecho de que una división territorial tenga una pequeña proporción de población dedicada a manufacturas puede indicar una especialización funcional y no un retraso en el desarrollo. Sin embargo, debe observarse que la diferenciación ocupacional dentro de las industrias constituye un indicador de desarrollo económico ya sea a nivel nacional o intranacional. Ciertamente que la especialización territorial puede alcanzar el punto donde ésta sea reflejada en la estructura ocupacional de una industria, pero se presume que México no ha alcanzado aún ese punto.

Los comentarios anteriores no deben interpretarse como sugerencias de que el desarrollo económico se refleja sólo a través de diferenciación ocupacional intraindustrial. Este se refleja también por la diferenciación industrial (esto es, sin considerar las ocupaciones) y por la diferenciación ocupacional (o sea, sin considerar las industrias). Si así fuera, la medida utilizada podría aplicarse a todos los elementos del cuadro 1; en este caso expresaría el monto total de diferenciación en las ocupaciones e industrias. De modo que el uso presente de esta medida constituye sólo una de varias alternativas en el análisis de la relación entre la diferenciación y el desarrollo.

Relaciones entre las medidas intraindustriales. La meta final de la investigación sobre división del trabajo no es, por supuesto, simplemente identificar las poblaciones como desarrolladas o subdesarrolladas. Por el contrario, dicha investigación pretende una explicación de las variaciones en la diferenciación ocupacional, y a ese respecto, las relaciones por entidades entre los ocho valores intraindustriales para $D O$ son de especial importancia. Si se encuentran relaciones estrechas y directas, es factible pensar en una explicación en términos de un factor, como eficiencia tecnológica total, o alguna medida correspondiente a la noción de "densidad" de Durkheim. Los datos del cuadro 3 cuestionan la idea de que cualquier explicación monista sea factible. Aun. que la mayoría de los coeficientes del cuadro son positivos, en conjunto la variación estatal en diferenciación ocupacional en una industria no 
Cuadro 2

Mí́xico: MEdidas de DIFERENCIACIÓN OCUPACIONAL INTRAINDUSTRIAL POR ENTIDADES, 1960 a

\begin{tabular}{|c|c|c|c|c|c|c|c|c|}
\hline & $\underset{\text { tura }}{\text { Agricul }}$ & Mineria & $\begin{array}{c}\text { Manufac } \\
\text { turas }\end{array}$ & Construccion & $\begin{array}{c}\text { Hectricidad, } \\
\text { gas, eto. }\end{array}$ & Comercio & $\underset{\text { teB }}{\text { Transpoz }}$ & Servicios \\
\hline Aguascalientes & .017 & .413 & .452 & .459 & .638 & .314 & .644 & .689 \\
\hline Baja California Norte & .052 & .525 & .477 & .499 & .664 & .459 & .468 & .727 \\
\hline Baja California Sur & .020 & .262 & .376 & .513 & .578 & .327 & .434 & .711 \\
\hline Campeche & .023 & .514 & .302 & .426 & .643 & .298 & .543 & .713 \\
\hline Coahuila & .033 & .232 & .385 & .524 & .663 & .437 & .608 & .683 \\
\hline Colima & .026 & .346 & .332 & & & .296 & .514 & .648 \\
\hline Chiapas & .016 & .482 & .247 & .487 & .653 & .262 & .543 & .641 \\
\hline Chihuahua & .033 & .267 & .456 & .541 & .657 & .441 & .547 & .685 \\
\hline Distrito Federal & .302 & .676 & .526 & .517 & .699 & .431 & .539 & .685 \\
\hline Durango & .022 & .230 & .407 & .506 & .626 & .330 & .559 & .706 \\
\hline Guanajuato & .023 & .377 & .247 & .411 & .727 & .184 & .530 & .702 \\
\hline Guerraro & .019 & .331 & .250 & .426 & .670 & .187 & .404 & .652 \\
\hline Hidalgo & .023 & .218 & .311 & .462 & .676 & .217 & & .603 \\
\hline Jalisco & .030 & .566 & .369 & .437 & .658 & .272 & .518 & .691 \\
\hline Néxico & .028 & .283 & .357 & .392 & .627 & .191 & .392 & .697 \\
\hline Michoacán & .021 & .370 & .233 & .402 & .682 & .163 & .350 & .667 \\
\hline Morelos & .027 & .544 & .475 & .441 & .660 & .249 & .397 & .656 \\
\hline Nayarit & .018 & .340 & & .378 & .624 & .177 & .443 & .730 \\
\hline Nuevo Lebn & .040 & .586 & .466 & .480 & .730 & .373 & .542 & .714 \\
\hline Oaraca & .015 & .389 & .389 & .424 & .685 & .178 & .603 & .614 \\
\hline Puebla & .021 & .522 & .265 & .407 & .685 & .215 & .538 & .657 \\
\hline Quare taro & .029 & .369 & .324 & .483 & .647 & .218 & .523 & .640 \\
\hline Quintina Roo & .015 & .722 & .285 & .398 & .577 & .324 & .526 & .717 \\
\hline San Iuis Potosi & .021 & .279 & .409 & .525 & .672 & .261 & .627 & .673 \\
\hline Sinaloa & .023 & .514 & .391 & .457 & .668 & . 296 & .466 & .743 \\
\hline Sonora & .045 & & .419 & .447 & .669 & .375 & & .727 \\
\hline Tabasco & .020 & .425 & .325 & .473 & .662 & .240 & .493 & .725 \\
\hline Tamaulipas & .027 & .547 & $.360^{\circ}$ & .414 & .651 & .314 & .503 & .706 \\
\hline Traxcala & .023 & .405 & .214 & .388 & .719 & .133 & .565 & .684 \\
\hline Veracruz & .021 & .351 & .316 & .436 & .640 & .259 & .576 & .669 \\
\hline $\begin{array}{l}\text { Yucatén } \\
\text { Zacatecag }\end{array}$ & .031 & . .461 & $\begin{array}{r}.262 \\
.250\end{array}$ & .372 & .651 & .226 & .557 & .729 \\
\hline Zacatecas & .014 & .176 & .250 & .432 & .624 & .186 & .475 & .723 \\
\hline $\begin{array}{l}\text { Valor más al to } \\
\text { Valor más bojo }\end{array}$ & $\begin{array}{l}.302 \\
.014\end{array}$ & $\begin{array}{l}.722 \\
.176\end{array}$ & $\begin{array}{l}.526 \\
.214\end{array}$ & $\begin{array}{l}.541 \\
.372\end{array}$ & .730 & .459 & .644 & $\begin{array}{l}.743 \\
.603\end{array}$ \\
\hline & & & & & & & & \\
\hline
\end{tabular}

a Véase en el texto la explicación de la medida de diferenciación ocupacional intraindustrial. 
está estrechamente asociada a la variación correspondiente en otra industria.

Cuadro 3

MÉXICO: COEFICIENTES DE CORRELACIÓN PRODLCTO-MOMENTO ENTRE MEDIDAS DE DIFERENCIACIÓN OCUPACIONAL INTRAINDUSTRIAL POR ENTIDADES, 1950 Y $1960^{\text {a }}$

\begin{tabular}{|c|c|c|c|c|c|c|c|c|}
\hline \multirow[b]{2}{*}{ Industria } & \multicolumn{3}{|r|}{ I } & $\begin{array}{lll}\mathbf{d} & \mathbf{u} & \mathbf{s}\end{array}$ & \multicolumn{2}{|c|}{ i $\mathbf{a}$} & & \multirow[b]{2}{*}{ Servicios } \\
\hline & $\begin{array}{l}\text { Agricul } \\
\text { tura }\end{array}$ & Mineria & $\begin{array}{c}\text { Manufac } \\
\text { turas }\end{array}$ & $\begin{array}{l}\text { Construe } \\
\text { ofón }\end{array}$ & $\begin{array}{l}\text { Utilis } \\
\text { dades }\end{array}$ & Comercio & $\begin{array}{l}\text { Trang } \\
\text { portes }\end{array}$ & \\
\hline $\begin{array}{l}\text { Agricultura } \\
\text { Mineria } \\
\text { Manufacturas } \\
\text { Conatruccion } \\
\text { Utilidades } \\
\text { Comercio } \\
\text { Transportes } \\
\text { Servicios }\end{array}$ & $\begin{array}{l}.38 \\
.46 \\
.30 \\
.26 \\
.42 \\
.07 \\
.03\end{array}$ & $\begin{array}{r}.41 \\
.20 \\
-.18 \\
.16 \\
.23 \\
.09 \\
.20\end{array}$ & $\begin{array}{l}.44 \\
.02 \\
.64 \\
.06 \\
.75 \\
.20 \\
.11\end{array}$ & $\begin{array}{r}.03 \\
-.20 \\
.042 \\
.02 \\
.68 \\
.27 \\
-.05\end{array}$ & $\begin{array}{r}.30 \\
.06 \\
.60 \\
.28 \\
-.10 \\
.14 \\
-.22\end{array}$ & $\begin{array}{l}.47 \\
.07 \\
.52 \\
.49 \\
.41 \\
.29 \\
.30\end{array}$ & $\begin{array}{r}.02 \\
-.08 \\
.14 \\
.06 \\
.08 \\
-.14 \\
.01\end{array}$ & $\begin{array}{r}.25 \\
.06 \\
.15 \\
.14 \\
.10 \\
.59 \\
-.40\end{array}$ \\
\hline
\end{tabular}

a $N$ es 32 para cada coeficiente; las cifras de 1950 quedan por encima de la diagonal y las de 1960 por debajo de ésta.

El cuadro 3 es tanto más importante porque un estudio previo (Gibbs y Browning, 1966) mostró esencialmente el mismo patrón en el plano internacional. Existe pues evidencia de que el desarrollo económico es divergente a nivel intranacional no solo territorialmente sino también entre industrias.

\section{TAMAÑ̃ RELATIVO DE LAS INDUSTRIAS}

Los coeficientes de correlación del cuadro 3 sugieren que no existe una característica sola de una entidad mexicana que pueda explicar la cantidad de diferenciación ocupacional en cada una de las industrias de la entidad, al menos porque los valores de $D O$ no varían congruentemente de una industria a otra. De acuerdo con esto, la explicación más probable es alguna propiedad de cada industria en cada entidad. Sería presuntuoso tratar de "explicar" o identificar la causa de la diferenciación ocupacional intraindustrial. Puede haber varios factores pertinentes (o "causas"), pero la presente investigación está limitada a la consideración de uno solo de los correlativos.

Dados los resultados presentados en el cuadro 3 , una propiedad de las industrias no tiene acción explicativa a menos que varíe de entidad a entidad para la misma industria y entre las diversas industrias dentro de una misma entidad. Una de dichas propiedades es el tamaño de la industria, lo cual se indicará a través del número de personas económicamente activas ocupadas en esa industria. Hay por lo menos dos razones para esperar que el tamaño de la industria esté relacionado en alguna forma con la diferenciación ocupacional intraindustrial. La primera idea (esencialmente de Durkheim) es que un aumento en el tamaño de una industria intensifica la competencia dentro de esa rama industrial, lo cual sólo puede solucionarse a través de la división del trabajo; por lo tanto, se esperaría encontrar una relación directa entre el tamaño de la industria y la diferenciación ocupacional 
intraindustrial. El segundo razonamiento toma en cuenta la evidencia de que las asociaciones altamente productivas fomentan la división del trabajo (Gibbs y Browning, 1966). El argumento puede ser extendido a la postulación de una relación directa entre el tamaño de una industria y el tamaño promedio de asociaciones productivas en esa industria. De acuerdo a lo anterior, si se acepta el argumento y su extensión, debería existir relación directa entre el tamaño de una industria y la diferenciación ocupacional intraindustrial.

E1 problema principal al examinar la relación es la medición del tamaño de las industrias. En el caso de las entidades mexicanas, el tamaño en cualquier industria dada tiende a ser en gran medida función del tamaño total de la población, el cual a su vez está determinado principalmente por la extensión de los límites de la entidad. Los límites son en general arbitrarios, ya que no delimitan unidades "reales" económicas o ecológicas. En consecuencia, una correlación por entidades entre el número de individuos empleados en una industria (es decir, el tamaño absoluto) y la diferenciación ocupacional no sería particularmente significativa. Una medida más justificable del tamaño de una industria es la proporción del total de la población económicamente activa de la entidad en esa industria, esto es, el tamaño relativo de la industria.

La columna 1 del cuadro 4 muestra la correlación por entidades mexicanas (1950) entre el tamaño relativo de una industria y el valor de $D O$ para la industria. La relación directa anticipada claramente no se da para algunas industrias y esto también es válido en 1960 (véase

\section{Cuadro 4}

MÉxico: COMPaRación dE dOS Relaciones POR ENTIDADES, CON RESPECTO A OCHO INDUSTRIAS, 1950

\begin{tabular}{|c|c|c|c|c|}
\hline \multicolumn{5}{|c|}{ Coeficiontes de correlacion (r) por entidades } \\
\hline & \multicolumn{2}{|c|}{$\begin{array}{l}\text { Entro diferenciación ocupacional } \\
\text { intraindustrial y proporcion } \\
\text { do la fuerza de trabajo } \\
\text { industrial on la ontidad } \\
\end{array}$} & \multicolumn{2}{|c|}{$\begin{array}{c}\text { Intre la proporción de la fuersa de } \\
\text { trabajo industrial on la entidad } \\
\text { y la proporción de la poblaoiba de } \\
\text { la entidad en looalidades } \\
\text { urbanas a }\end{array}$} \\
\hline & $\begin{array}{c}\text { Valor } \\
\text { (1) }\end{array}$ & $\begin{array}{c}\text { Rango } \\
(2)\end{array}$ & $\begin{array}{c}\text { Valox } \\
(3)\end{array}$ & $\underset{(4)}{\text { Rango }}$ \\
\hline $\begin{array}{l}\text { Agricul tura } \\
\text { Kinerta } \\
\text { fianuacturas } \\
\text { Construoció } \\
\text { Utilidades } \\
\text { Comercio } \\
\text { Transportes } \\
\text { Servicios }\end{array}$ & $\begin{array}{r}-0.84 \\
-0.55 \\
0.36 \\
0.08 \\
0.50 \\
0.52 \\
0.07 \\
0.44\end{array}$ & $\begin{array}{l}8 \\
7 \\
4 \\
5 \\
2 \\
1 \\
6 \\
3\end{array}$ & $\begin{array}{l}-0.90 \\
-0.05 \\
0.80 \\
0.89 \\
0.84 \\
0.92 \\
0.80 \\
0.75\end{array}$ & $\begin{array}{l}8 \\
7 \\
4 \cdot 5 \\
2 \\
3 \\
1 \\
4 \cdot 5 \\
6\end{array}$ \\
\hline
\end{tabular}

a Localidades urbanas con más de 2499 habitantes.

b Cada coeficiente está basado en un $N$ de 32 entidades.

la columna 1 del cuadro 5). Desde luego que en dos industrias (agricultura y minería) la relación es marcadamente inversa. Sin embargo, dada la notable variación tanto en la dirección como en la magnitud de los coeficientes de correlación, sería discutible concluir que simple- 
mente no existe relación. Al parecer, existe alguna clase de relación pero ésta es muy compleja. Las variaciones en los coeficientes de correlación claramente sugieren que la relación entre la división intraindustrial del trabajo y el tamaño relativo de una industria depende

Cuadro 5

MÉXICO: COMPARACión DE DOS RELACIONES POR ENTIDADES, CON RESPECTO A OCHO INDUSTRIAS, 1960

\begin{tabular}{|c|c|c|c|c|}
\hline \multicolumn{5}{|c|}{ Coolicientes de corrsacibn (x) por ontidedes } \\
\hline & \multicolumn{2}{|c|}{$\begin{array}{l}\text { Entro diferenciacion ocupacional } \\
\text { intraindustrial y proporoion } \\
\text { de la fueraa do trabajo } \\
\text { industrial on la entidad } \\
\end{array}$} & \multicolumn{2}{|c|}{$\begin{array}{l}\text { Entre la proporción de la fuerza de } \\
\text { trabajo industrial on la ontidad } \\
\text { J la proporoion de la poblacion de } \\
\text { la entidad on looalidados } \\
\text { urbanas a }\end{array}$} \\
\hline & (1) $^{\text {Valo }}$ & $\begin{array}{l}\text { Rango } \\
\text { (2) }\end{array}$ & $\begin{array}{c}\text { Valor } \\
\text { (3) }\end{array}$ & $\begin{array}{c}\operatorname{Rango} \\
(4)^{\circ}\end{array}$ \\
\hline $\begin{array}{l}\text { Agricul tura } \\
\text { Minerfa } \\
\text { Manufac turas } \\
\text { Cons trucctón } \\
\text { Utilidades } \\
\text { Comeroio } \\
\text { Transporte } \\
\text { Servioioa }\end{array}$ & $\begin{array}{l}-0.74 \\
-0.63 \\
0.51 \\
0.38 \\
0.09 \\
0.68 \\
0.24 \\
0.23\end{array}$ & $\begin{array}{l}8 \\
7 \\
2 \\
3 \\
6 \\
1 \\
4 \\
5\end{array}$ & $\begin{array}{r}-0.92 \\
-0.08 \\
0.81 \\
0.87 \\
0.82 \\
0.91 \\
0.84 \\
0.82\end{array}$ & $\begin{array}{l}8 \\
7 \\
6 \\
2 \\
4.5 \\
1 \\
3 \\
4.5\end{array}$ \\
\hline
\end{tabular}

a Localidades urbanas con más de 2499 habitantes.

b Cada coeficiente está basado en un $N$ de 32 entidades.

de una o más variables. Además podría ser que esta posibilidad cambiara en el tiempo, y que el cambio fuera mayor para unas industrias que para otras. Al comparar la columna 1 del cuadro 4 con la columna 1 del cuadro 5 se observa que algunos de los coeficientes de correlación son considerablemente diferentes en los dos momentos considerados (1950 y 1960). No obstante, las diferencias mayores son entre industrias en ambas fechas, más que cambios en las relaciones entre 1950 y 1960 . De acuerdo a lo anterior, en este punto el problema consiste en explicar la variación en la dirección y magnitud de la relación entre una industria y otra.

Concentración industrial. Una posibilidad es que el tamaño de una industria no tenga relación necesaria con la "densidad" de esa industria, consideración que nos vincula con el trabajo de Durkheim sobre la división del trabajo. Sin embargo, la teoría de Durkheim es ambigua y discutible. Dudó constantemente entre densidad "física o material" y densidad "social o moral" como causa de la división del trabajo. Si la densidad "física o material" (número de residentes por milla cuadrada) se toma como variable independiente, aun las observaciones más casuales pueden poner en duda la teoría. Específicamente, si algo existe, parece ser una ligera relación inversa entre la división del trabajo y la densidad de población a nivel internacional (compárese con las cifras en Gibbs y Martin, 1966). Pero si la densidad "social o moral" se toma como variable independiente, la teoría de Durkheim no es comparable porque es difícil imaginar un método de medida, o más 
aún conseguir los datos requeridos. Además, mientras que Durkheim presentó un argumento convincente para establecer una relación directa entre la densidad "física o material" y la división del trabajo (con competencia como la variable intermedia), no hay explicación particular en el caso de densidad "social o moral". De hecho, podría argumentarse que dicha densidad es efecto y no causa de la división del trabajo.

Durkheim empleó una terminología desafortunada para expresar su teoría. Si hubiera usado considerablemente el término "concentración" en vez de "densidad" la teoría hubiera sido menos ambigua y más consistente con las diferencias internacionales. Huelga decir que densidad y concentración no son conceptos intercambiables. La concentración no se refiere al número de residentes por unidad de área, sino al contrario, a la variación en ese número entre divisiones territoriales. Así, es lógicamente posible que una unidad territorial tenga una densidad total de tantos miles por milla cuadrada sin que exista concentración. Tendría concentración siempre y cuando esa densidad variara de una división a otra en la unidad territorial.

La concentración territorial es una propiedad muy importante de una industria, específicamente en relación con el tamaño relativo de la misma. La división del trabajo requiere algo más que grandes números; requiere también que los individuos empleados en una industria estén en contacto de modo que coordinen sus actividades. La concentración territorial de individuos en la misma industria hace más factible el contacto entre ellos porque reduce la fricción de espacio, esto es, la energía requerida para comunicarse, transportar materiales e intercambiar servicios. La idea general es que el tamaño de una industria fomenta la división del trabajo siempre y cuando los individuos empleados en esa industria estén concentrados. El Planteamiento IV establece la idea en términos formales y más empíricos: cuanto más concentrados territorialmente estén los individuos de una industria, más directa es la relación entre el tamaño relativo de la industria y la división del trabajo en esa industria.

El planteamiento IV introduce una variable descuidada en ecología humana y en economía - las bases territoriales de la división del trabajo. No es difícil ver por qué la investigación en este campo ha dejado mucho que desear. Dicha investigación es en extremo laboriosa y lograr comparabilidad internacional es un problema bastante complicado. Existen datos industriales para unos 2377 municipios en México y eventualmente puede ser posible computar una medida directa de concentración territorial para cada industria. Sin embargo, los recursos requeridos exceden en mucho los de la presente investigación. Por lo tanto, la concentración industrial se analiza indirectamente.

Un análisis indirecto de la concentración industrial considera la asociación entre el tamaño relativo de una industria y el grado de urbanización. La hipótesis es que una relación directa entre las dos variables refleja concentración territorial de la industria, como se establece formalmente en el Planteamiento V: cuanto más directa sea la relación entre el tamaño relativo de una industria y el grado de urbanización, habrá mayor concentración territorial de individuos en esa industria. El planteamiento $\mathrm{V}$ no es meramente verdadero por de- 
finición. Como ejemplo, supóngase que el coeficiente de correlación en países o entidades entre la proporción de individuos en la industria manufacturera y el grado de urbanización es 90. Dicho coeficiente sugiere que los trabajadores en manufacturas viven en áreas urbanas, lo cual es un indicador de concentración de la fuerza de trabajo. Sin embargo, si la asociación entraña la falacia llamada "correlación ecológica", entonces los inåividuos empleados en la industria manufacturera podrían ser residentes rurales predominantemente y por lo tanto están dispersos territorialmente. Por consiguiente, el supuesto que apoya el planteamiento $\mathrm{V}$ es que las correlaciones individuales y agregadas entre industria y lugar de residencia (rural o urbana) son congruentes. La correlación entre el tamaño relativo de una industria y el grado de urbanización podría aproximarse a +1.00 en un caso y a -1.00 en otro. Esta última posibilidad es importante, ya que sugiere que en algunos casos el tamaño relativo de una industria puede estar inversamente relacionado con la diferenciación ocupacional dentro de la industria. Dicha relación es paradójica, a menos que se considere la idea de Smith sobre el tamaño del mercado y la división del trabajo. En términos generales, su argumento es que un mercado grande facilita la división del trabajo.

El tamaño efectivo del mercado para algunas industrias puede ser en gran medida función del grado de urbanización de la región (entidades, en el caso de México). Si la industria produce principalmente para un mercado regional, entonces un alto grado de urbanización en una región significa un mercado concentrado. Ya que la concentración reduce la fricción de espacio, aumenta el tamaño efectivo del mercado. De acuerdo con esto, en algunas industrias, podría ser que el tamaño relativo de la industria variara inversamente con la diferenciación ocupacional debido a que su tamaño relativo varíe inversamente con el grado de urbanización.

Representación gráfica. La línea anterior de razonamiento puede ser ilustrada si nos referimos a la gráfica 1.

Obsérvese que los miembros de la industria $Z$ (agricultura) no están concentrados territorialmente; así que de una región a otra la proporción de la fuerza de trabajo en esa industria varía inversamente con el grado de urbanización, lo cual quiere decir que la relación inversa refleja la dispersión territorial de la industria. Como tal, podría ser que un aumento en el tamaño relativo de la industria no tuviera influencia sobre la división del trabajo en la industria. Sin embargo, si la industria produce principalmente para un mercado urbano en la región, el tamaño del mercado es función del grado de urbanización en la región. Suponiendo que un mercado grande facilita la división del trabajo (Smith) sin tomar en consideración el tamaño relativo de la industria $Z$ : tamaño relativo pequeño, alto grado de urbanización en la región, mercado grande, alto grado de división del trabajo dentro de la industria. Replanteado en términos más empíricos, la diferenciación ocupacional dentro de la industria $Z$ varia inversamente con respecto a la proporción de la fuerza de trabajo de la región que está en la industria. 
Gráfica 1

MODELO INTRAINDUSTRIAL DE DIFERENCIACIÓN OCUPACIONAL

Región $I$

\begin{tabular}{|lcccc|}
\hline$z_{1}$ & $Y_{1}$ & $z_{1}$ & $r_{1}$ & $z_{1}$ \\
$z_{1}$ & & $x_{1} x_{1} x_{2} x_{2}$ & & $z_{1}$ \\
$z_{1}$ & & $Y_{2}$ & $z_{1}$ \\
$z_{1}$ & & $Y_{1}$ & $z_{1}$ & $z_{1}$ \\
\hline
\end{tabular}

Región II

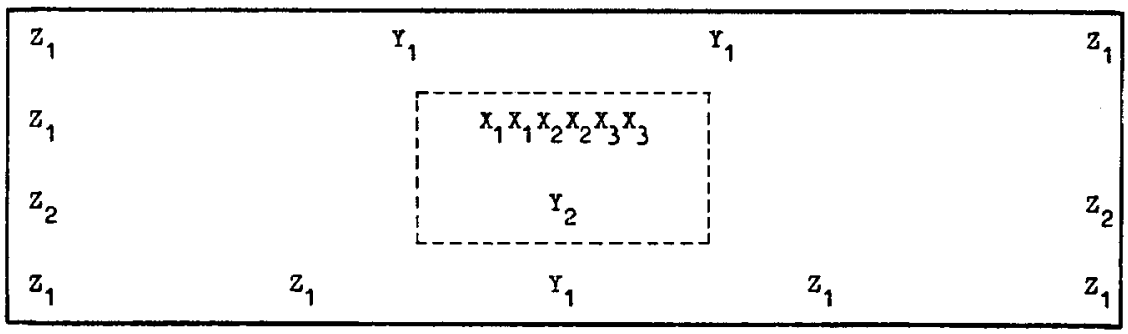

Region III

\begin{tabular}{|lcr|}
\hline$z_{1}$ & $x_{1}$ & $y_{1}$ \\
$z_{2}$ & $z_{3}$ \\
$z_{2}$ & $x_{1} x_{1} x_{2} x_{2} x_{3} x_{3} x_{4} x_{5}$ & $z_{2}$ \\
$y_{2}$ & $y_{1}$ & $z_{1}$ \\
\hline
\end{tabular}

Cada letra y número representa un miembro residente de la fuerza de trabajo. La letra significa una industria y el número significa una ocupación dentro de la industria.

Límites:

región ; -..- territorio urbano

En el caso de la industria $X$ (manufacturas), obsérvese que su ta. maño relativo varía directamente entre las regiones según el grado de urbanización, lo cual refleja la concentración territorial de la industria. Como tal, un aumento en el tamaño relativo de la industria facilita la división del trabajo pero esto sólo sucede cuando la industria está concentrada. Esa asociación se refleja en una relación directa en las regiones entre la proporción de la fuerza de trabajo regional en la industria $X$ y la diferenciación ocupacional en la industria. 
La industria $Y$ (algunos tipos de minería) es intermedia en el sentido que no está $\tan$ concentrada como la $X$ ni tan dispersa como la $Z$. De aquí que el tamaño relativo de $Y$ en una región no está estrechamente relacionado con el grado de urbanización en la región. Además, cuando el tamaño relativo de la industria es constante entre las regiones (como en la gráfica 1), no estará correlacionado con la diferenciación ocupacional, la cual también sería más o menos constante entre las regiones. Aun si el tamaño relativo de la industria debiera variar regionalmente, la diferenciación ocupacional podría permanecer más o menos constante de una región a otra. Como previamente se sugirió, siempre que una industria esté territorialmente dispersa, un aumento en su tamaño relativo puede no facilitar la división del trabajo, al menos no apreciablemente.

La única variable en el planteamiento $\mathrm{V}$ que no se ha considerado previamente es el grado de urbanización. Para las entidades mexicanas el grado de urbanización es designado como la proporción de la población total de la entidad en localidades de $\mathbf{2 5 0 0}$ habitantes y más. Los coefícientes de correlación por entidad entre el grado de urbanización y el tamaño relativo de cada industria se muestran en la columna 3 del cuadro 4 (1950) y en la columna 3 del cuadro 5 (1960). Al examinar los coeficientes se debe tener presente que los valores son tratados como indicadores de concentración territorial de una industria, esto es, cuanto más positivo sea el coeficiente para una industria, más concentrada territorialmente estará esa industria. En conjunto, los coeficientes son congruentes con las observaciones generales sobre concentración territorial de las industrias. De este modo, la agricultura tiende a ser una industria ubicua en México (en la gran mayoría de entidades territoriales de México una proporción considerable de la fuerza de trabajo está empleada en esa actividad), de aquí que no deba extrañar encontrar una relación inversa muy estrecha entre la proporción de personas empleadas en la agricultura y el grado de urbanización.

De las ocho industrias, el coeficiente de correlación es más discutible en el caso de la minería. A diferencia de la agricultura, la minería se lleva a cabo sólo en ciertos puntos. Sin embargo, se observa que la minería rara vez está asociada con grandes puntos de concentración de población; de aquí la baja correlación entre la minería y el grado de urbanización. Sin embargo, el hecho de que el coeficiente no varíe marcadamente en algunas industrias plantea algunas dudas. Específicamente, la ligera variación puede no revelar adecuadamente las diferencias en la concentración territorial de las industrias, pero esa duda no puede ser aclarada hasta que los recursos permitan una medida directa de la concentración territorial para cada industria en cada entidad.

Puede surgir una interpretación alternativa de los coeficientes en la columna 3 del cuadro 4 y el cuadro 5. Se podría argumentar que estos coeficientes reflejan únicamente el hecho de que algunas industrias son más "urbanas" que otras. El argumento es correcto pero no dice nada acerca de la importancia de caracterizar una industria como "urbana" o "rural". La importancia se encuentra en el contexto de la 
concentración territorial; una industria "urbana" es una industria "concentrada" y la concentración facilita la división del trabajo.

Dados los planteamientos III, IV y V, la derivación es el Planteamiento VI : cuanto más directa sea la relación entre el tamaño relativo de una industria y el grado de urbanización, más directa será la relación entre el tamaño relativo de la industria y la diferenciación ocupacional intraindustrial. El planteamiento VI nos lleva a la predicción de una relación directa entre los dos juegos de coeficientes de correlación en el cuadro 4 y también entre los dos juegos del cuadro 5 . Se aprecia que la noción de una "correlación entre correlaciones" es complicada y por esa razón se emplearon planteamientos formales para hacer explícito el argumento de base. La complejidad del fenómeno es tal que una relación simple y universalmente variable entre las dos variables es sumamente improbable. En este caso, es improbable que la relación entre dos propiedades cualquiera de una industria (por ejemplo, el tamaño relativo y la diferenciación ocupacional) permanezca invariable en todas las industrias. Como tal, el reto que se nos presenta es explicar la variación en una relación dada de una industria a la otra, y de aquí la consideración de una "relación entre relaciones".

La predicción directa de la relación entre los dos juegos de coeficientes de correlación fue probada computando dos valores rho -uno para el cuadro 4 y otro para el cuadro 5 . La $R$ ho es .74 para el primero y .76 para el segundo. De aquí que en las dos fechas consideradas la "relación entre relaciones" es como se anticipó; esto es, aceptando los coeficientes en la columna 3 de los cuadros 4 y 5 como indicativos de concentración territorial de una industria, cuanto mayor sea la concentración territorial de una industria, más directa es la relación entre diferenciación ocupacional y el tamaño relativo de la industria.

\section{RESUMEN Y CONCLUSTONES}

De acuerdo con lo esperado, la diferenciación ocupacional en una industria varía considerablemente entre las entidades mexicanas, un hecho que se interpreta como evidencia de tasas divergentes de desarrollo económico intranacional. Sin embargo, la diferenciación ocupacional en una industria no está estrechamente relacionada con el tamaño relativo de esa industria (la proporción de la fuerza de trabajo de la entidad en esa industria). La interpretación es que el tamaño relativo de una industria fomenta la división del trabajo siempre que la fuerza de trabajo de la industria esté concentrada territorialmente. Se presentó evidencia para confirmar esa interpretación tratando la correlación por entidades entre el tamaño relativo de una industria y el grado de urbanización como indicador de concentración territorial de esa industria. Los resultados son compatibles con la interpretación. Esto es, si el tamaño relativo de una industria está directamente relacionado con el grado de urbanización, está también directamente asociado al grado de diferenciación ocupacional en la industria.

Pudiera parecer que tanto los argumentos como los resultados son abstractos y que están relacionados escasamente con la tarea del fomento del desarrollo económico. Pero éste no es el caso. Si la meta de la política gubernamental es incrementar la división del trabajo en 
BROWNING/GIBBS: DIVISIÓN INTRAINDUSTRIAL DEL TRABAJO 303

las industrias, un método es fomentar la concentración territorial de las industrias a través digamos de una política fiscal diferencial por localización, o de subsidios directos a las industrias en ciertas partes del país. Esa observación implica el reconocimiento de que el fenómeno ecológico puede ser influido por políticas, lo cual implica decisiones conscientes y deliberadas. Sin embargo, de aquí no se concluye que las políticas, las decisiones, los valores, etc., no estén ellos mismos determinados por las condiciones ecológicas; $y$, sobre todo, que hay siempre límites a lo que puede lograrse a través de políticas. En el presente caso, por ejemplo, la concentración territorial de las industrias aumenta la distancia entre las instalaciones de una industria y las materias primas o el mercado. En cualquier caso, la adaptación favorable al aumento en distancia requiere una tecnología eficiente, especialmente en lo que toca a los transportes y las comunicaciones. De aquí que mientras se supone que la concentración territorial de las industrias pueda fomentarse a través de políticas, se reconoce que existen límites definidos a lo que esas políticas puedan lograr.

\section{REFERENCIAS BIBLIOGRÁFICAS}

Bataillon, Claude, Las regiones geográficas en México, México, Siglo XXI Editores, 1969.

Durkheim, Emile, The Division of Labor in Society, Glencoe, Illinois, The Free Press, 1949.

Gibbs, Jack P., y Walter T. Martin, "Urbanization, Technology, and the Division of Labor: International Patterns", American Sociological Review, 27, 1962 pp. 667-677.

Gibbs, Jack P., y Harley L. Browning, "The Division of Labor, Technology, and the Organization of Production in Twelve Countries", American Sociological Review, 31, 1966, pp. 81-92.

Hirschman, Albert O., The Strategy of Economic Development, New Haven, Yale University Press, 1958.

Hawley, Amos H., Human Ecology, Nueva York, Ronald Press, 1950.

Herskovits, Melville J., Economic Anthropology, Nueva York, Alfred A. Knopf, 1952.

Higgins, Benjamin, Economic Development, Nueva York, W. W. Norton, 1968.

Mir, Adolfo, Ecological Inequalities in Educational Attainment in Mexico, Tesis doctoral, inédita, University of Texas at Austin, 1970.

Smith, Adam, An Inquiry Into the Nature and Causes of the Wealth of Nations, Nueva York, Random House, 1937.

Unikel, Luis, "El proceso de urbanización", en El perfil de México en 1980, México, Siglo XXI Editores, 1970, Vol. 2, pp. 221-253.

Yates, Paul Lamartine, El desarrollo regional de México, México, Banco de México, 1961. 